\title{
A Hybrid Framework for Segmentation of MR Medical Images Using Adjusted Ant Colony Optimization \\ ${ }^{* 1}$ Yogesh Sayajirao Bahendwar, ${ }^{2}$ Dr. Rajesh H. Talwekar \\ ${ }^{1}$ S. B. Patil College of Engineering, Indapur, Maharashtra \\ ${ }^{2}$ Dr. Rajesh H. Talwekar, Government College of Engineering, Raipur, Chhattisgarh \\ Email: bahendwar72@gmail.com,ursk.talwekar@gmail.com
}

Received: 22 ${ }^{\text {nd }}$ December 2018, Accepted: 13 $^{\text {th }}$ February 2019, Published: $3^{\text {th }}$ June $^{2019}$

\begin{abstract}
In this paper a new framework is suggested for segmentation of medical images (MRI) using hybrid optimization technique. This frame work includes de-nosing as image enhancement technique using discrete wavelet transform and initial segmentation is obtained by Hidden Markov random field model along with Kmeans clustering to get Maximum a posterior values (thresholds). The optimal solution for these thresholds is achieved by incorporating a new concept of Adjusted Ant colony optimization. Finally a fine segmentation is obtained. The algorithm is tested for some of the medical images and for validation purpose various parameters like accuracy, sensitivity, dice, Jaccard, are obtained.
\end{abstract}

\section{Keywords}

De-Noising, Adjusted Ant Colony Optimization Algorithm (AACO), HMRF-EM, Ant Colony Optimization (ACO), Maximum a Posterior Values (MAP) Medical Image Processing, Computed Tomography (CT), Magnetic Resonance Imaging (MRI).

\section{Introduction}

Medical image segmentation is often the first step in quantitative image analysis. The aim of segmentation is to identify the target anatomy or pathology and delineate the boundary of these structures of interest. Image segmentation plays a vital role in numerous medical applications (Ahemadet al. 2006).Medical image analysis is a wide concept that includes several processing and analysis methods applied to a number of different imaging modalities. This image analysis can be divided into different subjects such as: Image enhancement, segmentation, registration, quantification.

Methods for performing segmentations vary widely, depending on the specific application, imaging modality, and other factors. For example, the segmentation of brain tissue has different requirements from the segmentation of the liver. General imaging artefacts such as noise, partial volume effects, and motion can also have significant consequences on the performance of segmentation algorithms. Furthermore, each imaging modality has its own idiosyncrasies with which to contend. There is currently no single segmentation method that yields acceptable results for every medical image. Methods do exist that are more general and can be applied to a variety of data. However, methods that are specialized to particular applications can often achieve better performance by taking into account prior knowledge. Selection of an appropriate approach to a segmentation problem can therefore be a difficult dilemma.

\section{Literature Review}

Aneja and Rawat (2013), proposed an algorithm for segmentation which performs de-noising using Fuzzy cmeans clustering. The performance of the proposed is assessed based on parameters like execution time, rate of convergence and cluster validity function and thus achieved MCR (Mis-classification rate) of $0.537 \%$ using IFCM (Intuitionistic fuzzy c-means) technique. Bedi and Goyal, (2010), reported about the development of noise filters to handle speckle noise in digital images. Prasad and Satheesh, (2011), proposed de-noising medical image using contour let transform algorithm.

Kumar et al.,(2010),Developed an improved method of segmentation using fuzzy-neurologic to detect different tissues for a given magnetic resonance image data set. Damodharan and Raghavan, (2015),effectively segmented given medical image for various tissues. They also have provided a comparative analysis for parameters like accuracy, specificity, and sensitivity that are obtained from various classifiers like K-Nearest Neighborhood (K-NN),Bayesian algorithm and their proposed technique based on neural network. They achieved an accuracy of $83 \%$ using neural network based classifier. Yang et al.,(2015) in their research proposed a technique for brain tumor clustering to cluster levoxel MR slices and obtained an accuracy and balance error rate as $94.2 \%$ and $7.8 \%$ respectively. Sachdevaet al., (2013, presented a multi class tumor classification, segmentation, and feature extraction and obtained classification accuracy from 77 to $91 \%$.Anitha et al., (2010), studied segmentation of lung lobes and nodules in computer tomography (CT) images. The 
algorithm was tested for CT images only. Ahemadet al., (2006) proposed the segmentation of brain MRI images for tumor extraction by combining k-means clustering and anisotropic diffusion method.

The rest of this paper is organized as follows. Section II discuss the related work, Section III describes the denoising process and later part deals with the segmentation of MR images using HMRF hybrid with AACO algorithm. Section IV compares the result of ACO, HMRF-ACO with the proposed HMRF hybrid with AACO. Section VI gives the conclusion for the paper.

\section{Materials and Methods}

Image De-Noising

The noise in the Medical image has random signals that are generated from machines and other sources in the environment but not from the tissue and do not participate in the random signal of tissue differentiation. This noise makes the image grainy and is distributed equally. The images can be destroyed with noise in two ways. One is by adding the noise image to the original image (additive noise), and the other is by multiplying the noise value with the original intensity (multiplicative noise). Wavelet shrinkage methods for de-noising images have been verified to implement the wavelets. The algorithm is summarized as

\section{Algorithm 1: Wavelet Image De-Noising}

- Select a wavelet (e.g. Haar, Symlet, etc) and decomposition levels or scales.

Calculate the forward wavelet transform of the noisy image.

- Estimating thresholds.

- Selecting the shrink rule and apply the threshold to the detail coefficients.

This can be done by hard thresholding

$$
\bar{c}_{h}(k)= \begin{cases}\operatorname{sign} c(k)(|c(k)|) & \text { if }|c(k)|>\lambda \\ 0 & \text { if }|c(k)| \leq \lambda\end{cases}
$$

or soft thresholding formula

$$
\bar{c}_{s}(k)= \begin{cases}\operatorname{sign} c(k)(|c(k)|-\lambda) & \text { if }|c(k)|>\lambda \\ 0 & \text { if }|c(k)| \leq \lambda\end{cases}
$$

- Apply an inverse transform (wavelet reconstruction) using the modified (threshold) coefficients.
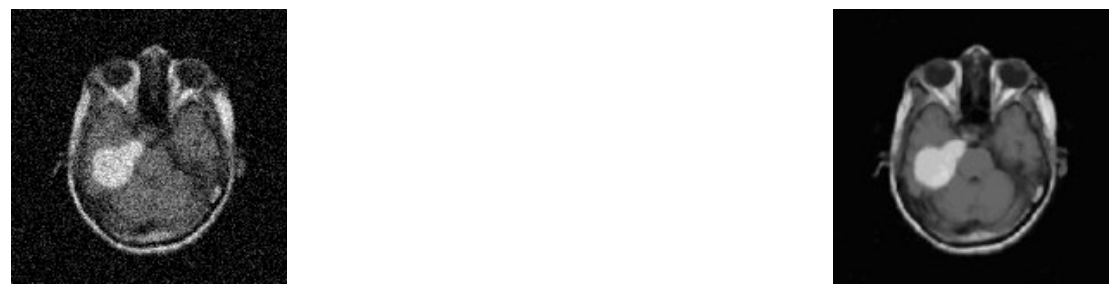

Figure 1: Noisy Image

Figure 2: Enhanced Image

\section{Segmentation Using HMRF-EM and AACO}

Generally, medical imaging is a term commonly used for technologies which are developed to create human images for medical use. The purpose of segmentation of Brain MRI image is to detect the suspicious area probably the tumor to assist physicians for diagnosis. Various methodologies like Fuzzy, region based and stochastic approaches are used in medical image segmentation techniques for segmentation of MRI images.

Edge detection is a traditional method for segmentation. Many operators like Roberts gradient, Sobel gradient, Prewitt gradient and Laplacian operator etc. can be used to detect the edges. Image segmentation can also be done using the morphological operations such as erosion, top-hat transformation etc. It is good in dealing with geometrically analytic aspects of image analysis problems.(Shelokaret al.2004;Dorigo et al, 2006; Liu \& tang 1999; He \& Chen 2001;Hongmin et al.,2007). Stochastic approaches have also been used to segment tumors. The watershed transformation for segmentation considers the gradient magnitude of an image as a topographic surface. Pixels having the highest gradient magnitude intensities correspond to watershed lines, which represent the region boundaries

Here, in this study we have proposed a system which deals with the noise present in the image during acquisition, performs segmentation and provides optimal results. The flow of the proposed system is 


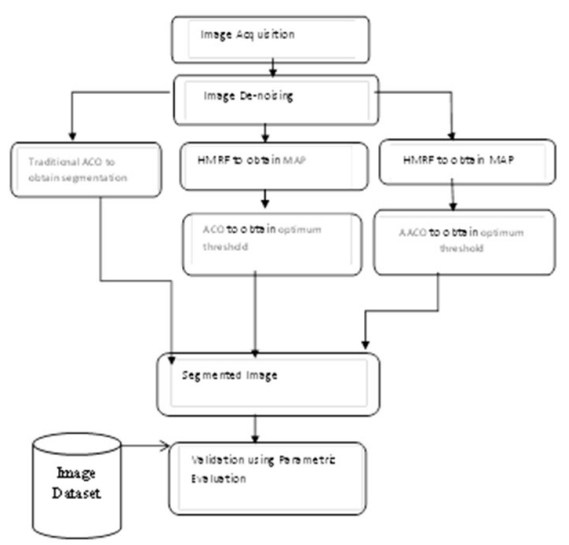

Figure Error! No text of specified style in document.: Segmentation Framework.

\section{HMRF-EM Model}

Hidden Markov Random Field (HMRF) model was used to deal with the spatial relations between the labels obtained in an iterative segmentation process. The HMRF is used to compute the MAP value of each kernel. The meta-heuristic algorithm Adjusted Ant colony optimization (AACO) is used to obtain the optimum labels by minimizing the MAP values. The central pixel intensity value is used as the threshold value for segmentation.

MRF technique is trusted in computer aide diagnosis for segmentation of medical images, reconstruction of inference and area, the HMRF algorithm is best suited to segmentation of images which works on strength of pixels. Some researchers modified or changed with the configuration of labels $\mathrm{X}=\left(\mathrm{x}_{1}, \ldots \ldots \ldots \mathrm{x}_{\mathrm{n}}\right)$

where $(x € \mathrm{~L})$ \& $\mathrm{L}$ denotes all possible labels.

The segmentation process changes with change in attributes like binary segmentation, $\mathrm{L}=\{0,1\}$, In the MAP criteria the label $\mathrm{X}^{*}$ may be expressed as

$\mathrm{X}^{*}=\operatorname{argmax}\{\mathrm{P}(\mathrm{y} \mid \mathrm{x}, \theta), \mathrm{p}(\mathrm{x})\}$

Here $\mathrm{p}(\mathrm{x})$ is Gibbs distribution (Wang 2012).

\section{EM Algorithm}

The parametric set $\theta$ is estimated by using by this algorithm.

It includes E-step and M-step.

The conditional expectations with all possible configuration of labels $\mathrm{Q}(\theta \mid \theta(\mathrm{t}))$ is calculated using E-step.

In M-step next estimate is obtained by maximizing $\mathrm{Q}(\theta \mid \theta(\mathrm{t}))$ as

$\Theta(\mathrm{t}+1)=\operatorname{argmax} Q(\Theta \mid \Theta(\mathrm{t}))$.

The Gaussian distribution function with parameter $\Theta \mathrm{l}=(\mu \mathrm{i}, \sigma \mathrm{l})$

$\mathrm{G}(\mathrm{z} ; \theta \mathrm{l})=\frac{1}{\sqrt{2 \pi^{2} \mathrm{l}}} \exp \left(-\frac{(\mathrm{z}-\mu \mathrm{l})^{2}}{2 \sigma^{2} \mathrm{l}}\right)$

And probability distribution

$\mathrm{P}(\mathrm{y} \mid \mathrm{x}, \theta)={ }_{\mathrm{z}}^{1} \exp (-\mathrm{U}(\mathrm{y} \mid \mathrm{x}))$

The HMRF-EM algorithm

1) Set initial parameter $\Theta(0)$

2) Calculate likely distribution $\mathrm{pt}$ )

3) Using parameter $\Theta(t)$, estimate the label by MAP estimation.

4) Update parameter using $\mathrm{p}(\mathrm{t})(1 \mid \mathrm{yi})$ with posteriori distribution for $1 € \mathrm{~L} \&$ all possible pixels yi(Wang 2012).

The initial labels are obtained by k-means clustering on grey scale intensity of pixels, for using HMRF-EM Algorithm. The initial parameter $\Theta(0)$ obtained for EM algorithm and initial labels $\mathrm{X}(0)$ for MAP algorithm. Refined segmentation is obtained on execution of EM algorithm.

\section{The Ant Colony Optimization (ACO) Algorithm}

Ant colony optimization is developed by M. Dorigo to solve the optimization problems. ACO is a nature inspired algorithm. The foraging behavior of ants is taken as a key feature to optimize the given problem. The communication strategy followed in ants based on depositing a special chemical discharge called pheromone. Each Ant searches for pheromone and after visit deposits its pheromone, in this way pheromone update occurs (Yanpeng 2007)The movement ants increases the concentration pheromone thereby increasing the probability 
of other ants to follow the same path. Due to evaporation the pheromone of other path decreases for other ants (Yongqiang 2003). Thus the entire colony finds and follows the shortest path from nest to the food source.

Ant colony optimization algorithm is suggested in combination with HMRF-EM, so that the optimal segmentation can be achieved. Usually, "food" is defined in the segmentation of images which gets memorized by a number of elements called Ants. The further motion of ants is governed by the pixels deposited by ants. The position of ants change in accordance with certain transition rules applied in iterative manner (Lee et al. 2003).

While splitting an image, it is necessary to find the characteristics which reflect the differences. Pixel gray value is used as a clustering feature as it greatly differ with the background and it normally mutate at boundary points and derivative of these points reflects the changes. In ACO each pixel in the image is treated as Ant and two dimensional vector is considered as corresponding pixel pheromone (Liu et al. 2014). Ants foraging behaviour based segmentation is process in which ants with different characteristics searches for food source. Ants stop roaming in the image, when encountered with pixel of dissimilar characteristics (i.e. pheromone feature).

For given image $\mathrm{K}$, the transition probability of a pixel i.e. ants $\mathrm{Ki}$ choosing to transfer to $\mathrm{Kj}$ is given by equation

$\mathrm{P}_{\mathrm{ij}}^{\mathrm{k}}=\left\{\begin{array}{c}\frac{\tau_{\mathrm{ij}}^{\alpha} \eta_{i j}^{\beta}}{\sum_{\mathrm{j} \in \mathrm{M}} \tau_{\mathrm{ij}}^{\alpha} \eta_{\mathrm{ij}}^{\beta}} \\ 0, \text { otherwise }\end{array}\right\} \mathrm{j} \varepsilon \mathrm{M}$

Where $\tau_{\mathrm{ij}}$ is the pheromone that ants carry with them; $\eta_{\mathrm{ij}}$ is the heuristic function; $\alpha$ and $\beta$ represents the pheromone accumulated and the percentage role of heuristic function, while moving a pixel to the cluster. M denotes the set of possible path for next step.

The Euclidiean distance between the pixel $\mathrm{Ki}$ to $\mathrm{Kj}$ is calculated as

$$
\begin{aligned}
& d_{i j}=\sqrt{\left(K_{i 1}-K_{j 1}\right)^{2}+\left(K_{i 2}-K_{j 2}\right)^{2}} \\
& \eta_{i j}=\frac{1}{d_{i j}}
\end{aligned}
$$

The transition rule is defined as

$\tau_{i j}(t+1)=(1-\rho) \tau_{i j}(t)+\rho \Delta \tau_{i j}$

Where $\rho$ is the pheromone evaporation coefficient $\Delta \tau_{i j}$, is the pheromone increment

$$
\Delta \tau_{i j}=\sum_{l=1}^{n} \Delta \tau_{i j}^{l}
$$

$\Delta \tau_{i j}^{l}$ is the amount of information that $l$-th ant leaves in the path $\mathrm{ij}$.

\section{Adjusted Ant colony Optimization (AACO)}

This section suggests an improved segmentation method with adaptive ant colony optimization. In the initial stage, we applied a Hidden Markov Random field model with k-means clustering which allows ants to collect quickly to get thresholds of the image. AACO is implemented to get optimal thresholds. As discussed in section 3.3 , in the final stage of construction process ant tends to death by arriving to one of its previous visited node or pixel. The death probability of ants can be avoided by using dynamic neighbourhood pixel probability. Thus the escape ration of ants is increased to $50 \%$ by taking 12 neighbouring pixels into calculations.

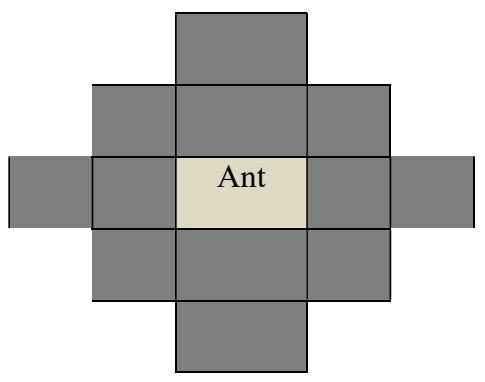

Figure 4: Search Clique Increased to 12 Pixels

\section{Proposed Algorithm}

Algorithm: HybridACO with HMRF-EM

The Steps involved in proposed framework are

1 Read 2D medical image (CT or X-ray or MRI) 
2. Convert the given image to Gray scale image

3. Obtain binary map $\mathrm{Z}$ and observable y using canny edge detection and K-means

4. Apply HMRF-EM segmentation method

5. Set initial parameter $\Theta^{(0)}$

6. Calculate Likely hood distribution $\mathrm{p}(\mathrm{t})$

7. Using Parameter $\theta^{(t)}$, estimate the label by MAP estimate

$\mathrm{X}^{(\mathrm{t})}=\operatorname{argmax}\{\mathrm{p}(\mathrm{y} \mid \mathrm{x}, \Theta), \mathrm{p}(\mathrm{x})\}$

$$
\begin{array}{ll}
=\operatorname{argmin}\left\{\mathrm{U}\left(\mathrm{y} \mid \mathrm{x}, \Theta^{(0)}\right)+\mathrm{U}(\mathrm{x})\right\} & \mathrm{X} \in \mathrm{x} \\
\mathrm{X} \in \mathrm{x}
\end{array}
$$

8. Calculate posterior distribution for all possible pixels $\mathrm{p}(\mathrm{t})$

9. Get the sum of posterior energy distribution for each iteration

Sum_U $=$ I to iterations

10. Repeat from 6 until converges

11. To minimize the posterior function $U(x)$ Ant colony optimization is used

12. Initialize parameters alpha ,beta, $\mathrm{T}(0)$, pheromone coefficient and Definition of neighbourhood clique 4 pixel or 8 pixel in traditional ACO.

13. Select initial pheromone value to 0.001

14. Select random pixel which is not selected yet and initialize position of ant.

15. Record position of ant.

16. Find the neighbourhood of current position based on search cliques definition.as in step 12 .

17 Here in our new approach (AACO) we adjusted search cliques for neighbourhood pixel as 12 pixels.

18. Calculate transition probability to the neighbourhood of current position.

19. Generate the random number for next position

20. Record the delta $\mathrm{p}$ of current position

21. Switch to new position \& update pheromone function.

22. Take the mean of thresholds above average and below average.

23. The stopping condition reaches if the two consecutive thresholds are same and/or iterations are over.

24. If condition satisfies in step 23 indicates there is no new edge further

Evaluation of Proposed Segmentation Framework

The performance of proposed framework can be evaluated with reference to the parameters such as Accuracy, Sensitivity, Jaccard and Dice. The confusion matrix that defines the terms TP,TN,FP,FN for ground truth results and probable outcome for calculating accuracy, sensitivity, Jaccard and Dice is shown in Table 1 Jaccard and Dice similarity index are also evaluated based on number of voxels of the ground truth and segmented image.

\begin{tabular}{|l|l|l|l|}
\hline Outcome (Expected) & \multicolumn{2}{|c|}{ Ground Truth } & \multicolumn{1}{c|}{ Total (Row) } \\
\hline & Negative & Positive & \\
\hline Negative & TN & FN & TN+FN \\
\hline Positive & FP & TP & TP+FP \\
\hline Total (Column) & TN+FP & FN+TP & TN+FN+TP+FP \\
\hline
\end{tabular}

Table 1: Confusion Matrix Showing Definition of terms TP, FP, TN and FN

\begin{tabular}{|l|l|}
\hline \multicolumn{1}{|c|}{ Evaluation Parameter } & \multicolumn{1}{c|}{ Formula } \\
\hline Accuracy & $(\mathrm{TP}+\mathrm{TN}) / \mathrm{N}$ \\
\hline Sensitivity & $\mathrm{TP} / \mathrm{p}$ \\
\hline Jaccard & Common/Union \\
\hline Average Dice Similarity Coefficient & $(2 *$ common $) /(\mathrm{cm}+\mathrm{co})$ \\
\hline
\end{tabular}

Table 2: Formulae's to Calculate Parameters

\section{Results and Discussion}

For experiment purpose online web based medical image database is used The HMRF-EM algorithm has been tested over 30 MRI images. A sample result for one of the MRI image has been shown in Figure 5, in which Fig.5 (a) is an Original image, Fig.5 (b) shows the result of existing Ant colony optimization.

For comparison purpose ACO is hybrid with HMRF-EM in Fig5(c) and Fig.5(d) result of improved hybrid AACO with HMRF-EM , The complete test procedure took 34.634 seconds on Intel Pentium $2.2 \mathrm{GHz}$ processor. 


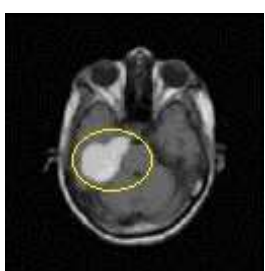

(a)

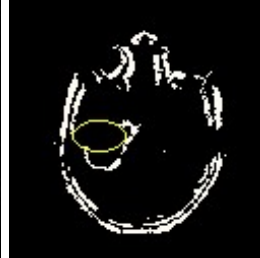

(b)

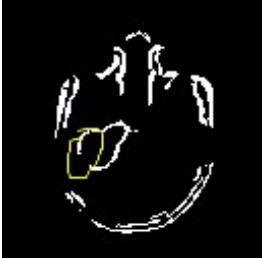

(c)

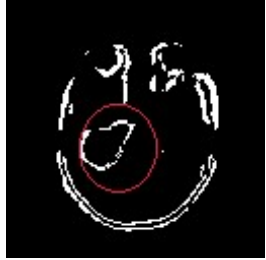

(d)

Figure 5: Segmentation Results, Fig.5 (a) is an Original Image; Fig.5 (b) Shows the Result of Existing ACO, in Fig 5(c) ACO Combined with HMRF-EM and Fig 5(d) Result of Proposed Improved Hybrid AACO with HMRF-EM.

\begin{tabular}{|c|l|l|l|}
\hline Parameters Assessed & $\begin{array}{l}\text { Traditional } \\
\text { ACO }\end{array}$ & $\begin{array}{l}\text { Hybrid Traditional ACO with } \\
\text { HMRF-EM (Proposed) }\end{array}$ & $\begin{array}{l}\text { Hybrid AACO with } \\
\text { HMRF-EM (Proposed) }\end{array}$ \\
\hline Accuracy & $79.5 \%$ & $84.2 \%$ & $96.2 \%$ \\
\hline sensitivity & $86.3 \%$ & $91.9 \%$ & $95.5 \%$ \\
\hline Jaccard & $82.9 \%$ & $92.1 \%$ & $96.1 \%$ \\
\hline $\begin{array}{c}\text { Dice Similarity } \\
\text { coefficient }\end{array}$ & $82.2 \%$ & $89.3 \%$ & $92.1 \%$ \\
\hline
\end{tabular}

Table 3: Comparative Analysis of Accuracy, sensitivity, Jaccard and Dice Similarity Coefficients.

\section{Conclusion}

In this paper, the meta-heuristic image segmentation approach is presented. The approach used for segmentation is the Ant colony optimization. The improved accuracy rate according to the experimental results is due to better classification of natural brain structure. Experiments on MRI images show that the segmentation result of the proposed method has higher accuracy compared to existing algorithms.

\section{Conflict of Interest}

The authors declare that there is no financial or third party conflict of interest in publishing this article.

\section{References}

[1] Ahemad M. Masroor and Mohamad Dzulkifli Bin, "Segmentation of Brain MR Images for Tumor Extraction by Combining K-means Clustering and Perona-Malik Anisotropic Diffusion Model". International Journal of Image Processing.2 (1):27-34,(2006).

[2] Aneja D and Rawat T. K, "Fuzzy clustering algorithms for effective medical image segmentation," International Journal of Intelligent Systems and Applications, vol. 5, 2013.

[3] Bedi C.S. and Goyal H. "Qualitative and Quantitative Evaluation of Image De-noising Techniques" International Journal of Computer Application”, vol 4 (14) pp 31-34, 2010.

[4] Satheesh S., Kvsvr Prasad. "Medical image denoising using adaptive threshold based on contour let transform.” Advanced Computing International Journal, 2(2):52-58, 2011.

[5] Kumar S S, Moorthi M, Madh M, Amutha R: An improved method of segmentation using fuzzyneuro logic. In: Proc: Second International Conference on Computer Research and Development, Kuala Lumpur, 2010, pp 671-675

[6] Damodharan S, Raghavan D: Combining tissue segmentation and neural network for brain tumor detection. Int Arab J InfTechnol 12:42-52, 2015.

[7] Yang G, Nawaz T, Barrick T R, Howe F A, Slabaugh G: Discrete wavelet transform-based wholespectral and subspectral analysis for improved brain tumor clustering using single voxel mr spectroscopy. IEEE Trans Biomed Eng 62:2860-2866, 2015

[8] Sachdeva J, Kumar V, Gupta I, Khandelwal N, Ahuja C K: Segmentation, feature extraction, and multiclass brain tumor classification. J Digit Imaging 26:1141-1150, 2013.

[9] Anitha S. and Sridhar S., Segmentation of Lung Lobes and Nodules in CT Images. Signal \& Image Processing : An International Journal. 1(1):1-12,2010.

[10] P.S. Shelokar, V.K. JayRaman and B.D. Kulkami, "An ant colony clustering approach for clustering”, ANALYTICAL CHMICALACTA 509, 2004, pp.187- 195.

[11] M. Dorigo, Mauro Birattari and Thomas Stiitzle, "Ant Colony Optimization artificial Ants as a Computational Intelligence Technique”, Computational Intelligence magazine, Nov. 2006, pp.28-39. 
[12] J. Liu, Y.Y. Tang, “Adaptive image segmentation with distributed behavior-based agents," IEEE Transactions Pattern Analysis and Machine Intelligence 21, June 1999, pp. 544-551.

[13] H. He, Y. Chen, “Artificial life for image segmentation,” International Journal of Pattern Recognition and Artificial Intelligence 15 (6),2001, pp. 989-1003.

[14]Hongmin Cai, Ragini Verma, angming Ou, Seung-koolee, Elias R.Melhem, Christos Davatzaikos,: "Probabilistic Segmentation of brain tumors Based on Multi-Modality Magnetic Resonance Images", IEEE, ISBI 2007

[15] Quan Wang., "HMRF-EM-Image: Implementation of Hidden Markov Random Field Model and its Expectation-Maximization Algorithm" arXiv:1207.3510v2[cS.cv] 18 dec 2012.

[16] L. Yanpeng, "Research on Ant Colony Optimization and Its Application", Zhejiang University, Zhejiang, (2007).

[17] C. Yongqiang, "Artificial ant colony algorithm and its application in combinatorial optimization", HeiLongjiang, Harbin Institute of Technology, (2003)

[18] Myung-eun Lee et al., "Segmentation of Brain MR Images using Ant colony optimization Algorithm" Ninth IEEE International conferece on Bioinformatics and BioEngineering" Taichung,22-24 Jun 2009.

[19] Xumin Liu, Xiaojun Wang, Na Shi and Cailing Li "Image Segmentation Algorithm Based on Improved Ant Colony Algorithm International journal of signal processing, image processing and pattern recognition Vol.7, No.3 (2014), pp.433-442. 